\title{
Recurrent Gallstone Ileus in an Elderly Patient With Significant Co-Morbidity
}

\author{
Andrejs Vanags*, Mara Sneidere*, Ilze Strumfa**, Zane Simtniece**, Maris Pavars*, Janis Gardovskis* \\ *Department of Surgery, Riga Stradins University, Latvia \\ ${ }^{* *}$ Department of Pathology, Riga Stradins University, Latvia
}

\section{Summary}

Gallstone ileus accounts for 1-4\% of mechanical bowel obstruction. As the patients are elderly, significant co-morbidity is frequent, therefore early diagnosis and timely surgical treatment is mandatory to prevent patient's death. The aim of the present case report is to heighten the awareness of surgeons to the occurrence and differential diagnostics of gallstone ileus. Here we describe recurrent intestinal obstruction in an elderly lady with previously treated peritonitis as well as history of hip joint prosthesis and mitral valve replacement.

Key words: calculous cholecystitis, cholecystoduodenal fistula, gallstone ileus, Bouveret syndrome, peritonitis

\section{AIM OF THE DEMONSTRATION}

Aim of the demonstration is to describe a rare surgical pathology in order to heighten the awareness of surgeons so that gallstone ileus can be included in the differential diagnostics of intestinal and gastric outlet obstruction.

\section{CASE REPORT}

Seventy-two-year-old female was urgently admitted to Clinical University hospital with severe pain in the umbilical region. The pain had lasted for 2 days. Following the onset of pain, bouts of vomiting had developed as well. Physical investigation revealed severe pain, abdominal wall rigidity and rebound pain by palpation. Intestinal peristalsis was absent. There were signs of deep dehydration. The patient had complicated medical history including 2 previous urgent hospital admissions within several months. Four months before the present episode, she had had prolonged abdominal pain. Destructive calculous cholecystitis was diagnosed. By laparoscopic approach, a perivesicular infiltrate was drained. Three months later, the patient was admitted to the hospital with severe abdominal pain. Obstructive ileus was revealed. By laparotomy approach, a stonelike object was removed from the intestines through enterotomy. Surgeon interpreted this object as a phitobezoar. In all previous cases, the surgical treatment was embarrassed by overdosed anticoagulant therapy due to hip joint and mitral valve prostheses. The hypocoagulation was corrected by fresh frozen plasma. Taking into account the patient's medical history, urgent computed tomography (CT) of abdomen with oral and intravenous contrast enhancement was performed. By $\mathrm{CT}$, infiltrate and abscess-like cavity measuring $3 \mathrm{~cm}$ was found in the subhepatic area (Fig.1). Exudate was found in the right abdominal flank and in pelvis. There were signs of ileus in small intestines as well (Fig.2).
Laboratory findings showed elevated C-reactive protein of $244.9 \mathrm{mg} / \mathrm{L}$ [laboratory reference interval 0-5.0 $\mathrm{mg} / \mathrm{L}]$, creatinine $118 \mathrm{mkmol} / \mathrm{L}$ [35-97 $\mathrm{mkmol} / \mathrm{L}]$, urea $10.2 \mathrm{mmol} / \mathrm{L}$ [2.0-7.1 $\mathrm{mmol} / \mathrm{L}]$, direct bilirubin $11 \mathrm{mkmol} / \mathrm{L}[<7 \mathrm{mkmol} / \mathrm{L}]$, ASAT $55 \mathrm{U} / \mathrm{I}[<41 \mathrm{U} / \mathrm{I}]$ and ALAT $58 \mathrm{U} / \mathrm{I}[<58 \mathrm{U} / \mathrm{I}]$. The level of alpha amylase in the urine was $1094 \mathrm{U} / \mathrm{I}[<460 \mathrm{U} / \mathrm{I}]$. Anticoagulants have been used before admission to the hospital, so patient had coagulation disorders reflected by the following findings: activated partial thromboplastin time $37.6 \mathrm{sec}$., international normalized ratio (INR) 2.5, fibrinogen 4.4 $\mathrm{g} / \mathrm{L}$ and prothrombin index $25.4 \%$. The white blood cell count was $4.0 \times 10^{9} / \mathrm{L}$; platelet count $125 \times 10^{9} / \mathrm{L}$ and red blood cell count $3.52 \times 10^{12} / \mathrm{L}$.

Based on clinical findings and imaging, ileus was treated conservatively for 1 day. As there was no positive dynamics, surgical therapy was applied. During laparotomy, diffuse stercoral peritonitis was found with fibrin-rich exudate. It was caused by hemicircular intestinal perforation located $160 \mathrm{~cm}$ proximally from ileocaecal valve, just before the previous enterotomy scar. A stone was impacted in the perforation. After the intestinal integrity was repaired, inspection of abdominal cavity disclosed $3 \mathrm{~cm}$ wide cholecystoduodenal fistula with perforation (Fig.3). The fistula was closed as well. Postoperatively patient was treated in intensive care unit for 3 days. Later, reaching haemodynamical stability, she was transferred to surgical department. Postoperative recovery was long and slow. Vancomycin was administered to eradicate methicillin-resistant Stapholococcus aureus. Hydrothorax was eliminated by puncture. After massive medical treatment for 5 weeks, eventually patient was discharged with improvement. No recurrences of peritonitis or acute abdominal pain of any reason have occurred during the following 5 months. 


\section{DISCUSSION}

Calculous cholecystitis is a frequent disease in Western world. The total amount of stones in the gall bladders in the population is estimated in tonnes. The presence of gallstones can lead to diverse complications. Biliary enteric fistula develops in $2-3 \%$ of patients with cholecystolithiasis and recurrent cholecystitis (Roslyn et al., 1987; Giese et al., 2010). If choledochoduodenal fistula has developed, the stones can pass into the small intestines and occasionally become trapped causing ileus. Gallstone ileus accounts for 1-4\% of mechanical bowel obstruction and in aged persons (older than 65 years) - for $25 \%$ of non-strangulated small bowel obstruction cases (Giese et al., 2010). Due to the sedimentation of intestinal content, gallstones can increase in diameter and more frequently impact in the distal part of small intestine (Giese et al., 2010). The impacted stone is located in the terminal ileum in $50-75 \%$, proximal ileum and jejunum in $20-40 \%$, and in duodenum in $10 \%$ cases. If gastric outlet obstruction develops due to gallstone impaction in the duodenum or pylorus, the condition is called Bouveret syndrome. It is a rare manifestation of gallstone ileus, caused mainly by large stone. Until 1999, only 175 cases of Bouveret syndrome were reported (Ariche et al., 1999).

In the diagnostics, history of recent gallstone disease exacerbation should alert attention. However, as in our case, the formation of fistula itself can occur during clinically silent period after the exacerbation (Giese et al., 2010). CT and ultrasonography can provide valuable information (Giese et al, 2010). However, occasionally the findings are pathological still ambiguous. Thus, in our patient cholecystoduodenal fistula was interpreted as an abscess. Presence of stone in the intestinal perforation must prompt intraoperative investigation of biliary system.

The treatment of gallstone ileus is aimed at removal of the obstructing stone. The treatment can include endoscopic methods, especially in case of proximal stone location as in Bouveret syndrome. Endoscopic extraction, extracorporeal shock wave lithotripsy or laser lithotripsy can be successful (Maiss et al., 2004; Tanwar et al., 2008). However, endoscopic treatment can fail if the stone is large (O'Neill et al., 2009). In addition, the fragments can undergo growth by sedimentation resulting in more distal stone impaction and recurrent ileus (O'Neill et al., 2009). Regarding surgery, it is recommended to remove the stone through enterotomy using either laparoscopic or open surgical approach, and leave the gallbladder and fistula in situ as this is associated with lower mortality and recurrent complications are rare (O'Neill et al., 2009). However, in our case, the course of disease was already notable for recurrences therefore restitution of intestinal integrity was followed by closure of the fistula during the same operation. The outcome was beneficial as the patient survived and experienced no further recurrences. Thus, our experience is more in line with the contrary suggestion of active surgical treatment and closure of fistula (Iancu et al., 2008).
Significant co-morbidities are described in gallstone ileus patients, including frequent consequences of atherosclerosis (O'Neill et al., 2009). The association can be attributed to common occurrence of both cholecystolithiasis and atherosclerotic heart and vessel disease in elderly group. In contrast, vascular pathology could enhance ischemic changes thus facilitating fistula formation. Regardless of pathogenesis, this association compromise the outcome and can become cause of death. Therefore the diagnosis should be reached early, before peritonitis has developed.

\section{Conflict of interest: None}

\section{REFERENCES}

1. Ariche A, Czeiger D, Gortzak Y, Shaked G, Shelef I, Levy I. Gastric outlet obstruction by gallstone: Bouveret syndrome // Scand J Gastroenterol, 1999; 35:781 - 783

2. Giese A, Zieren J, Winnekendonk G, Henning BF. Development of a duodenal gallstone ileus with gastric outlet obstruction (Bouveret syndrome) four months after successful treatment of symptomatic gallstone disease with cholecystitis and cholangitis: a case report // J Med Case Rep, 2010; 4:376

3. Iancu C, Bodea R, Hajjar N, Todea-Iancu D, Bala O, Acalovschi I. Bouveret syndrome associated with acute gangrenous cholecystitis // J Gastrointestin Liver Dis, 2008; 17(1):87 - 90

4. Maiss J, Hochberger J, Hahn EG, Lederer R, Schneider HT, Muehldorfer S. Successful laserlithotripsy in Bouveret's syndrome using a new frequency doubled doublepulse Nd:YAG laser (FREDDY) // Scand J Gastroenterol, 2004; 39 (8): 791 - 794

5. O'Neill C, Colquhoun P, Schlachta CM, EtemadRezai R, Jayaraman S. Gastric Outlet obstruction secondary to biliary calculi: 2 cases of Bouveret syndrome // Can J Surg, 2009; 52(1):E16 - 18

6. Roslyn J, Thompson JJ, Darvin H, DenBesten L.Risk factors for gallbladder perforation // Am J Gastroenterol, 1987; 82:636-640

7. Tanwar S, Mawas A, Tutton M, O'Riordan D. Successful endoscopic management of Bouveret's syndrome in a patient with cholecystoduodenocolic fistulae // Case Rep Gastroenterol, 2008; 2(3):346 350

\section{Address:}

Andrejs Vanags,

Department of Surgery,

Riga Stradins University

Pilsonu Street 13, LV 1002, Riga, Latvia

E-mail: vanags314@inbox.lv 


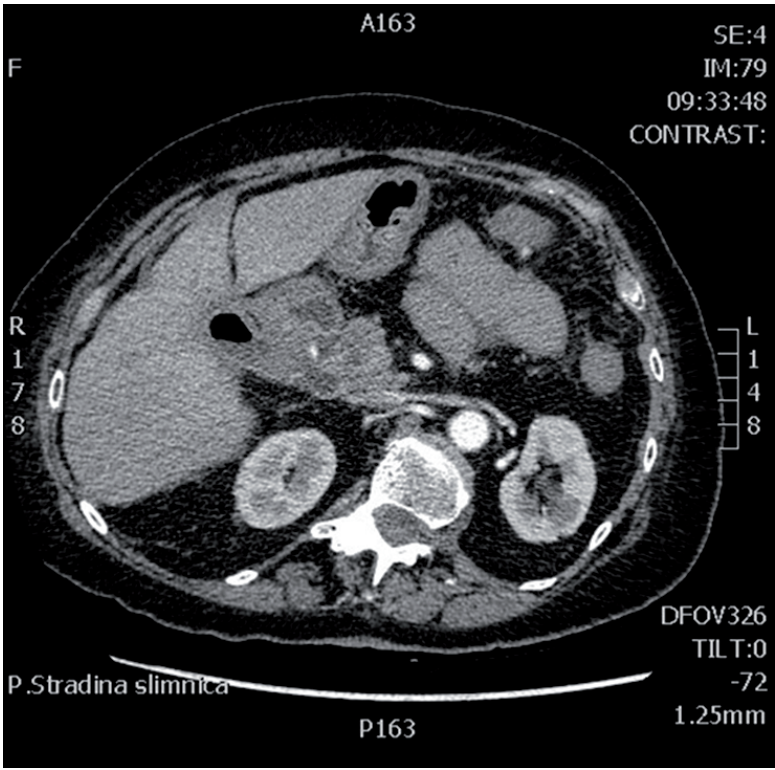

Fig. 1. Abdominal computed tomography findings before the operation. Note the subhepatic infiltrate and cavity.

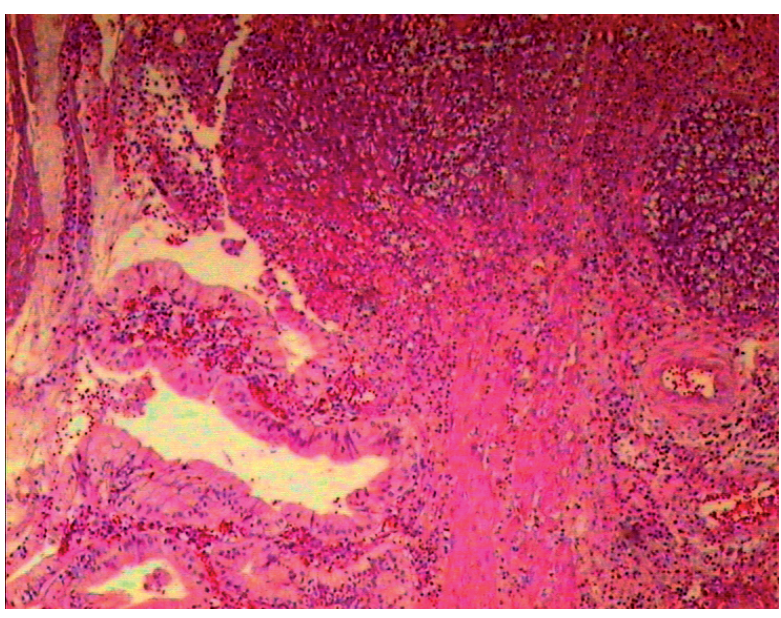

Fig. 3. Tissue structure of the resected holecystoduodenal fistula. Note the deep necrosis and purulent inflammation in the wall of fistula reflecting changes adjacent to fistula perforation site. Haematoxylin-eosin, original magnification 50x.

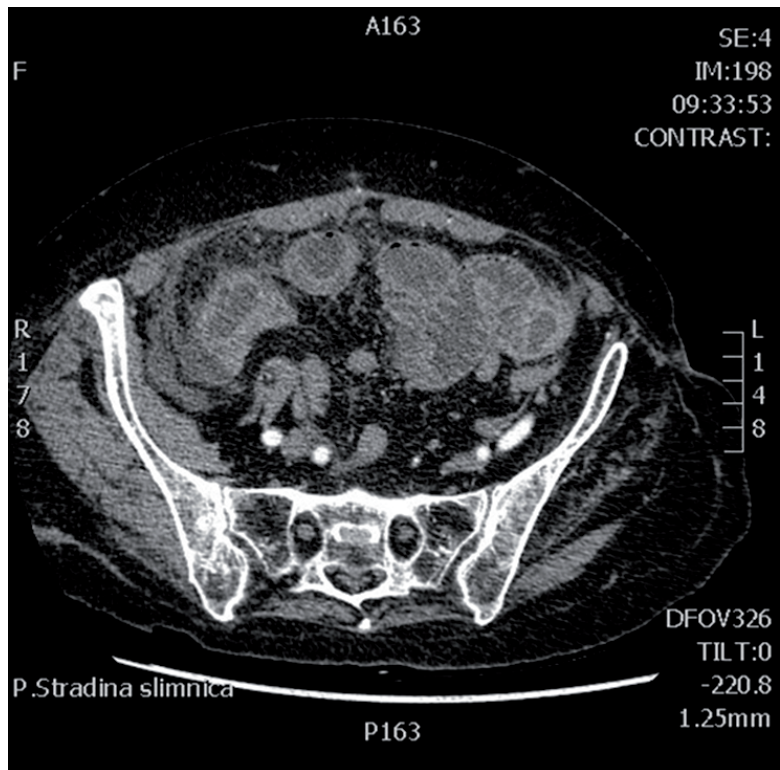

Fig. 2. Abdominal computed tomography findings before the operation. Note the markedly distended intestinal loops. 